\title{
CIDADE EDUCADORA: A DIMENSÃO CULTURAL NO PROCESSO IDENTITÁRIO DE SOLEDADE
}

\author{
CIUDAD EDUCADORA LA DIMENCIÓN CULTURAL EN EL PROCESO IDENTIFICATÓRIO DE \\ SOLEDADE
}

\author{
EDUCATING CITY: A CULTURAL DIMENSION IN THE IDENTITY PROCESS OF SOLEDADE \\ CITY
}

\author{
Gisele Lamaison de Freitas ${ }^{1}$ \\ Eliara Zavieruka Levinski²
}

\begin{abstract}
RESUMO
Esse estudo de natureza qualitativa e bibliográfica, objetiva refletir e compreender as contribuições da cultura dos cidadãos no processo educativo da Cidade Educadora, assim como socializar práticas pedagógicas desenvolvidas em espaços escolares e não escolares no município de Soledade. $O$ texto que ora apresentamos é um recorte do trabalho final do curso de Pós-graduação lato sensu Especialização Políticas e Gestão da Educação realizado in company entre a Universidade de Passo Fundo e a Prefeitura Municipal de Soledade. A cultura constitui-se nas relações estabelecidas entre as pessoas, revela concepções, modo de pensar e viver, costumes e valores. Também está associada à produção de atividades artísticas e o desenvolvimento social. É uma dimensão que integra a formação identitária dos povos. No decorrer da reflexão, pautaremos a concepção de cidade educadora, Soledade como Cidade Educadora, compreensões sobre a cultura como uma das categorias mobilizadoras da discussão com ênfase ao tradicionalismo e aos projetos e ações culturais que inscrevem a vida cotidiana da população soledadense. A cultura influencia na formação da identidade dos cidadãos por meio das instituições sociais e integra uma dimensão fundamental de um território educador.
\end{abstract}

PALAVRAS-CHAVE: Cultura. Cidade Educadora. Tradicionalismo. Práticas Pedagógicas.

\section{ABSTRACT}

This qualitative and bibliographic study aims to reflect and understand the contributions of citizens' culture to the educational process of Educating City, as well as to socialize pedagogical practices developed in school and non-school spaces in the municipality of Soledade. The text presented here is an excerpt from the final work of the Post-Graduation lato sensu Specialization Policies and Management of Education carried out in company between the University of Passo Fundo and the Municipality of Soledade. Culture is constituted in the relationships established between people, it reveals concepts, ways of thinking and living, customs and values. It is also associated with the production of artistic activities and social development. It is a dimension that integrates the identity formation of peoples. During the reflection we will guide the concept of an educating city, Soledade as an Educating City, understandings about culture as one of the categories that mobilize the discussion, with emphasis on traditionalism and cultural projects and actions, which inscribe the daily life of the population of Soledad. Culture influences the formation of citizens' identity through social institutions and integrates a fundamental dimension of an educating territory.

\footnotetext{
1 Graduada em Educação Física e Especialista em Políticas e Gestão da Educação pela Universidade de Passo Fundo, professora da Rede Estadual de Ensino do Rio Grande do Sulgiselelamaison@hotmail.com

2 Doutora em Educação pela Universidade Federal do Rio Grande do Sul. Integrante do Grupo de Pesquisa e Extensão Políticas e Gestão da Educação da Universidade de Passo Fundo - GPEPGE. eliarazavlev@gmail.com
} 
KEYWORDS: Culture. Educating City. Traditionalism. Pedagogical practices.

\section{RESUMEN}

Este estudio de naturaleza cualitativa y bibliográfica, tiene como objetivo la reflexión y compreención sobre el conocimiento que los ciudadanos puedan aportar culturalmente al proceso educativo de ciudad educadora, y también compartir practicas pedagógicas realizadas en espacios escolares y no escolares en el municipio de Soledade. El texto que a seguir presentamos es un recorte del trabajo final del curso de posgrado lato sensu Especialización Política y gestión de la Educación realizado conjuntamente entre la Universidad de Passo Fundo y la Intendencia Municipal de Soledade. La cultura de un pueblo se crea por las relaciones entre las personas, su forma de pensar, sus costumbres y sus valores. A esto se suman las diversas actividades astísticas y el intercambio social que realizan los ciudadanos. Todo esto sirve para mensurar la formación de la identidad de un pueblo. En el transcurso de nuestra reflexión trataremos sobre el concepto de ciudad educadora, sobre Soledade siendo Ciudad Educadora, entendimientos de los detalles de una cultura, discutiendo, com mayor dedicación y énfasis al movimiento denominado Tradicionalismo y los projectos y acciones culturales que dictan y nortean la vida cotidiana de la población soledadense. La cultura tiene su influéncia en la formación de la identidad de los ciudadanos através de las instituciones sociales y protagoniza una dimención fundamental de un territorio educador.

PALABRAS CLAVES: Cultura. Ciudad Educadora. Tradicionalismo. Prácticas Pedagógicas.

\section{INTRODUÇÃO}

As inquietações que mobilizaram este estudo de cunho bibliográfico estão ancoradas na experiência cidadã em movimentos da cultura gaúcha, nas práticas da profissão docente no ensino público de Soledade e nos momentos formativos da especialização Políticas e Gestão da Educação. Essas foram traduzidas na pergunta: em que medida a cultura territorial influencia na formação da identidade do povo por meio das instituições sociais que constituem uma cidade como educadora? Desse modo, desejamos refletir e compreender as contribuições da cultura no processo educativo da Cidade Educadora, assim como socializar práticas pedagógicas desenvolvidas em espaços escolares e não escolares no município de Soledade. Cultura e cidade são categorias que conectadas colaboram para compreensão da identidade do território.

Para a materialização dos objetivos e explicitação da pergunta de trabalho, abordaremos questões relacionadas à educação, cultura, cidade educadora, cultura gaúcha e tradicionalismo³, assim como a rede de espaços públicos que inscrevem Soledade na Associação Internacional de Cidades Educadoras (AICE) ${ }^{4}$.

\footnotetext{
${ }^{3}$ Movimento popular que visa auxiliar o Estado na consecução do bem coletivo, através de ações que 0 povo pratica (mesmo que não se aperceba de tal finalidade) com o fim de reforçar o núcleo de sua cultura: graças ao que a sociedade adquire maior tranquilidade na vida comum (LESSA, 1954, texto digital).

${ }^{4}$ Fundada em 1994, a Associação Internacional das Cidades Educadoras (AICE) é uma Associação sem fins lucrativos constituída como uma estrutura permanente de colaboração entre governos locais que se comprometem a reger-se pelos princípios inscritos na Carta das Cidades Educadoras. https://www.edcities.org/pt/quem-somos/
} 


\title{
CIDADE EDUCADORA: ALGUMAS REFERÊNCIAS
}

Enquanto educadora, a Cidade é também educanda. Paulo Freire

Com o passar dos tempos, a sociedade muda e outros processos educativos são constituídos. Há infinitas possibilidades de educar-se na e com a sociedade. A educação como ato humano acontece em todos os cantos, experimenta-se nas inter-relações e interações com 0 meio em diferentes contatos e com diferentes ações.

Para Brandão "ninguém escapa da educação, em casa, na rua, na igreja ou na escola, de um modo ou de muitos, todos nós envolvemos pedaços da vida com ela: para aprender, para ensinar, para aprender-e-ensinar todos os dias misturamos a vida com a educação". (2007, p.7)

Continuando a reflexão, Gómez-Granel e Vila, destacam que:

O esforço educativo não pode ser feito unicamente a partir da escola. A escola não tem e não pode ter sozinha a responsabilidade pela educação. Ela não pode responder indiscriminadamente a todas as demandas que lhe são feitas, nem tem de ocupar todos os momentos da vida dos alunos". (2003, p. 31)

Nessa linha, Brandão reitera e adensa o valor dos saberes dos sujeitos da comunidade:

\begin{abstract}
O saber da comunidade, aquilo que todos conhecem de algum modo; o saber próprio dos homens e das mulheres, de crianças, adolescentes, jovens, adultos e velhos; 0 saber de guerreiros e esposas; o saber que faz 0 artesão, o sacerdote, o feiticeiro, 0 navegador e outros tantos especialistas, envolve, portanto, situações pedagógicas interpessoais, familiares e comunitárias, em que ainda não surgiram técnicas pedagógicas escolares, acompanhadas de seus profissionais de aplicação exclusiva. (2007, p. 20)
\end{abstract}

Os autores congregam diretrizes de que a educação forma e transforma, que percorre a vida nos múltiplos tempos e territórios. No conjunto desse processo educativo, a escola, com sua função social de socializar o conhecimento que a humanidade acumulou historicamente e de colaborar para a formação cidadã, dialoga estreitamente com o seu território, com a sua cidade na medida em que aprende, tematiza e vivencia o lugar no seu currículo. Nesse contexto, Gadotti (2006) destaca que o papel da escola é contribuir para criar as condições que viabilizem a cidadania, por meio da socialização da informação, da discussão, da transparência, gerando uma nova mentalidade, uma nova cultura, em relação ao caráter público do espaço da cidade ( $p$. 136). Continua o autor, observando que: 
A escola educa para ouvir e respeitar as diferenças, a diversidade que compõe a cidade e que se constitui na sua grande riqueza. $O$ cidadão da cidade educadora presta atenção ao diferente e também ao "deficiente", ou melhor, ao portador de direitos especiais. Para que a escola seja espaço de vida e não de morte, ela precisa estar aberta para a diversidade cultural, étnica e de gênero, e às diferentes opções sexuais. As diferenças exigem uma nova escola. (GADOTTI, 2006, p. 138)

Assim, a escola e a cidade, interdependentemente, densificam os processos educativos que formam e transformam os sujeitos do lugar. Com essa compreensão, a educação é associada à cidade educadora, pois ao mesmo tempo a cidade educa e é educada. Freire afirma que:

\begin{abstract}
A cidade converte-se em cidade educadora a partir da necessidade de educar, de aprender, de imaginar...; sendo educadora, a cidade é, por sua vez, educada. Uma boa parte da sua tarefa como educadora está ligada ao nosso posicionamento político e, obviamente, ao modo como exercemos o poder na cidade e ao sonho e utopia que impregnam a nossa política, no serviço do que e de quem servimos œ a política de despesa pública, a política cultural e educativa, a política de saúde, transporte e lazer. (1992, p. 25-27)
\end{abstract}

Uma cidade educadora deve ser educadora em todos os espaços, com todos os setores, com todos os sujeitos que nela moram, deverá favorecer, entre outros, a liberdade de expressão, a diversidade cultural e o diálogo em condições de igualdade, bem como entre gerações. $\mathrm{Na}$ cidade educadora, as diferentes políticas, espaços, tempos e atores são compreendidos como agentes pedagógicos, capazes de apoiar o desenvolvimento de todo potencial humano. Nas palavras de Levinski; Tascheto (2013), a cidade torna-se educadora quando decide e assume intersetorialmente o objetivo e a responsabilidade da formação, promoção e desenvolvimento de todos os seus munícipes.

Com essa perspectiva, compreende-se que a educação das crianças, jovens e cidadãos em geral não é somente responsabilidade das instituições tradicionais (estado, família e escola), mas também deve ser assumida pelo município, por associações, instituições culturais, empresas com vontade educadora e por todas as instâncias da sociedade.

Segundo Cabezudo (2004), hoje mais do que nunca, a cidade grande ou pequena dispõe de incontáveis possibilidades educadoras. Cada território, com seu jeito singular, pode intencionar e realizar múltiplas interlocuções com a sua e outras regiões, com o objetivo de possibilitar práticas educativas que promovam a formação e desenvolvimento humano com igualdade e justiça social. 
O potencial educador das cidades nem sempre é reconhecido pelos sujeitos e representantes da cidade e, consequentemente, essa dimensão fica ausente nos projetos, programas e políticas públicas do território.

Nesse sentido, faz-se necessário considerar um olhar sobre os processos educativos que a cidade constitui com sua população e sobre as concepções de cidade educadora, para desenhar um projeto de gestão orientado pela participação social e pelo direito à cidade.

\section{SITUANDO SOLEDADE COMO CIDADE EDUCADORA}

Soledade, localizada no Alto da Serra do Botucaraí, no norte Gaúcho, aproximadamente 200 quilômetros de Porto Alegre, possui 31 mil habitantes. Com vastas áreas rurais e uma topografia rica em pedras preciosas, o município é também rico em lendas regionais que preservam a cultura local na cidade centenária e desde que surgiu o resgate da cultura territorial no Rio Grande do Sul, entre as décadas de 40 e 50, o município vem estabelecendo uma forte caraterística de manifestação cultural, através da atividade artística, literária, recreativa ou esportiva, que caracteriza o Tradicionalismo, num esforço permanente de entrega e amor à cultura gaúcha, que perpassa gerações integrando os indivíduos até os dias atuais.

A capital das Pedras Preciosas também foi considerada e muitos ainda a consideram como a Cidade Mais Autêntica do Rio Grande do Sul e esse título sempre será um desafio para gestão municipal e tradicionalistas no sentido de manter, através da cultura, o desenvolvimento da comunidade, estabelecendo um processo formativo permanente, consciente e integrador.

Soledade é também a primeira cidade da região norte gaúcha e a $16^{\mathrm{a}}$ brasileira a entrar para a lista internacional de Cidades Educadoras da AICE (Associação Internacional de Cidades Educadoras). A gênese do processo de buscar o reconhecimento de cidade educadora está assentada, primeiramente, na formação continuada dos profissionais da educação que, ao refletirem sobre a função social da escola, ramificam as discussões sobre o currículo e a interlocução com os segmentos da comunidade. Com a assessoria do Centro Regional de Educação, da Universidade de Passo Fundo (UPF), desde 2014, o Sistema Municipal de Ensino intensificou 0 capital formativo com ênfase aos processos democráticos e a mobilização para interrogações e estudos acerca da cidade que temos e queremos. 
A participação de Soledade nos eventos promovidos pelo Programa de extensão "UniverCidade Educadora"5, da UPF, que abordaram a temática de Cidades Educadoras e Inteligentes, é mais um indicador que agregou saberes e motivações para ampliar o debate em reuniões com o executivo, legislativo e segmentos da sociedade e entidades não governamentais para apresentação do real significado das cidades educadoras e quais compromissos gerariam para posterior decisão de tornar-se membro da Associação Internacional das Cidades Educadoras.

No processo de indagação sobre que cidade somos e que cidade ainda podemos ser, os segmentos foram mobilizados a localizarem práticas e desafios existentes no território que pudessem justificar o desejo de Soledade tornar-se cidade educadora, pois um lugar não se torna ou é identificado como educador somente com ações potencializadoras, mas pelo modo como pensa, lida e faz a sua própria cidade, o território não é emblemado como educador somente por realizar atividades benéficas à população, mas principalmente pelo modo como vivencia a própria cidade ancorada nos princípios que buscam a qualidade de vida, a garantia do direito à cidade e à humanização.

Essa perspectiva necessita de permanente reflexão por parte dos governantes e da população, na relação com o modo de estar constituindo a cidade, tornando-se pauta central dos projetos e ações que mobilizam a gestão do território.

Em uma das suas reflexões, Craidy destaca que falar da cidade como ambiente educativo é um desafio.

\footnotetext{
Uma cidade educadora não apenas terá escolas que possibilitem boas condições de vida e de saber para os que as frequentam, mas criará espaços de participação nas diferentes dimensões da vida cotidiana: da cultura e do lazer às formas de oportunidades de trabalho. As exigências educativas se impõem, sobretudo, na condução do próprio processo de gestão da cidade, que será tanto mais educativo, quanto mais democrático. (2002, p. 13)
}

Entre os desafios da cidade educadora está a valorização dos seus costumes e suas origens e a socialização dos saberes construídos em diferentes espaços, sejam eles escolares e não escolares.

5 O Programa UniverCidade Educadora integra a extensão da Universidade de Passo Fundo/RS. Constitui-se de uma estratégia de promoção de práticas de cidades educadoras e inteligentes no Município de Passo Fundo/RS e Região. 


\section{A CULTURA GAÚCHA NA CIDADE EDUCADORA DE SOLEDADE: UM RESGATE DO MOVIMENTO}

Para Carlos Rodrigues Brandão, "cultura é tudo aquilo que criamos a partir do que nos é dado, quando tomamos as coisas da natureza e recriamos como os objetos utensílios da vida social" (2002, p. 22).

A cidade, feita de cantos e momentos diversos, construídos e inventados no dia a dia tem como motivação constante a promoção e o exercício educador na vida dos sujeitos, por meio da construção de cidades mais inclusivas, mais justas e mais participativas, com especial destaque para a elaboração de ferramentas que oportunizem a todos o exercício pleno de sua cidadania. Os espaços públicos, instituições sociais formais e não formais, que acolhem e abrem suas portas para fomentar a cultura regional, as diferentes artes e manifestações culturais colaboram no processo educativo dos cidadãos.

Soledade tem, na sua identidade cultural, a presença da cultura gaúcha, com mais ênfase no tradicionalismo. As manifestações estão presentes nas escolas, Centro de Tradições Gaúchas- CTG, Centros Culturais, Praças Públicas, Parque de Evento Rui Ortiz, Museu da Pedra e Mineralogia Egisto Dal Santo.

A participação de crianças e jovens na rotina escolar apresenta um universo de sociabilidade. Entende-se que a função social da escola é a construção de saberes historicamente construídos com o objetivo principal de formar cidadãos críticos e participativos na sociedade, devendo esta ser um espaço de valorização e estimulação da participação dos estudantes de forma organizada e promotora de princípios. Quando a escola passa a ter significado na vida da cidade ou da comunidade, constituirá sentido para os alunos, impulsionando-os ao protagonismo e liderança nos espaços da vida cotidiana.

Nos espaços escolares, os Grêmios Estudantis mobilizam, juntamente com outras práticas escolares, para a formação cidadã. [...] cumpriram sempre um importante papel na formação e no desenvolvimento educacional, cultural e esportivo da nossa juventude [...]. As atividades dos Grêmios Estudantis representam para muitos jovens os primeiros passos na vida social, cultural e política (PARANÁ, 2012, p. 7) e no caso aqui, no Rio Grande do Sul, esta agremiação teve papel fundamental no resgate e na preservação da nossa identidade cultural.

O Sistema Municipal de Ensino de Soledade, com atuação em espaços formais e não formais, desenvolve processos com estratégias que fomentam a cultura gaúcha. Através da 
atividade artística, literária, recreativa ou esportiva, o Tradicionalismo procura, mais que tudo, reforçar o núcleo da cultura rio-grandense.

Nesse sentido, Lessa (1954) ressalta que o tradicionalismo é um movimento que age dentro da psicologia coletiva. Sua dinâmica realiza-se por intermédio dos Centros de Tradições Gaúchas, agremiações de cunho popular que têm por fim estudar, divulgar e fazer com que 0 povo "viva" as tradições rio-grandenses.

A escola, como mais um espaço educativo, agrega potencialidades para promover a cultura gaúcha, oportunizando conhecimento e experiências e, consequentemente, ramificando 0 legado nas comunidades.

O escritor gaúcho Barbosa Lessa, em sua Tese O Sentido e o Valor do Tradicionalismo, aprovado pelo Primeiro Congresso Tradicionalista do Rio Grande do Sul, deixa registrado que 0 Movimento Tradicionalista Rio-grandense eclodiu forte, em agosto de 1947, com a proposta de esperança, de liberdade e o amor à terra. Jovens estudantes oriundos do meio rural, de todas as classes sociais, liderados por Paixão Côrtes, criam um Departamento de Tradições Gaúchas, movimento este nascido do Grêmio Estudantil do Colégio Júlio de Castilhos de Porto Alegre.

Com a finalidade de preservar as tradições gaúchas, mas também de desenvolver e proporcionar uma revitalização da cultura rio-grandense, interligando-se e valorizando no contexto da cultura brasileira, esse movimento deu origem ao Grupo dos Oito que, na sequência, formou os Centros de Tradições Gaúchas. Os estudantes do Grêmio Estudantil destacaram a importância desse processo ter lugar formativo nos espaços escolares. Os professores primários do Rio Grande do Sul - conscientes do sentido profundo desse gesto, e não por simples atitude de simpatia, ofereceram apoio a iniciativa cultural.

Nesse movimento, algumas escolas do município de Soledade, na década de 50, criaram invernadas artísticas dentro do espaço escolar. Dessa forma, os alunos tiveram a oportunidade de vivenciar a cultura gaúcha, dando os primeiros passos para que 20 anos depois as escolas se entrelaçassem com a entidade tradicionalista.

Em setembro de 1973, nas comemorações da Semana Farroupilha, o Patrão Carlito Borges Lamaison, do Centro de Tradições Gaúchas Marciano Brum (fundado em 16.04.1953), sendo a primeira entidade da $14^{\mathrm{a}}$ Região Tradicionalista e $17^{\mathrm{a}}$ a ser fundada no estado, convidou as escolas para se apresentarem pela primeira vez na sede da entidade. A primeira edição teve a participação de somente duas escolas, sendo elas: Escola Maria de Abreu e Lima, coordenada pela "Tia Doli" e a Escola Medianeira- Coordenada pelas Irmãs Capuchinhas. Dessa forma, a cultura territorial estabeleceu um vínculo oficial e determinante entre as escolas locais e o CTG 
no Município de Soledade. Nos anos seguintes, foram instituídos os concursos de Danças Tradicionais e individuais motivados pelos tradicionalistas Airton Jacob da Silva Brum- 0 Carreteiro e Vilson Silva de Freitas. Esse projeto estimulou a adesão de alunos na sua maioria oriundos das escolas municipais. Assim, surgiu um dos maiores movimentos culturais de Soledade que é fortemente cultuado até os dias atuais.

Destaca-se, nesse percurso, a partir dos trabalhos realizados com alunos e coordenados pela professora Sandra Soldatelli, a criação do Grupo de Arte Nativa Vaqueanos da Cultura, em abril de 1979, na Escola Estadual Polivalente. O processo de fundação do Grupo foi conduzido e acompanhado pelo casal tradicionalista Vilson Silva de Freitas e Margarete Lamaison de Freitas. O Grupo com suas manifestações é reconhecido nos âmbitos estadual e internacional, alargando a dimensão da cultura de Soledade.

Outras entidades foram criadas, uma vertente cultural que se espalhou pelo município em diferentes bairros que se entrelaçaram com as escolas, criando uma teia associativa de cultura e participação social, um processo educacional que curricularizou intencionalidades, projetos e múltiplas atividades educacionais.

O município de Soledade é reconhecido para além da própria região que pertence como lugar que preserva e potencializa da cultura gaúcha.

\section{ESPAÇOS PÚBLICOS: REDE DE MANIFESTAÇÕES DA CULTURA}

Os espaços públicos congregam potencial para múltiplas oportunidades educativas e são campos formativos. Para Gehl, (2004) A humanização do espaço público e sua ocupação como lugar de formação são fundamentais para que a cidade se torne um lugar gerador de pertencimento e cidadania. Cultura e educação são conceitos que dialogam e estabelecem uma interlocução na vida cotidiana da população, transversalizam a mobilidade, transporte, segurança, meio ambiente, inscrevem a cidade.

O desafio é fazer um processo de gestão intersetorial, em cenários que apontam para práticas isoladas e sem enlaces com a integralidade da vida. O movimento para tal desafio implica na participação dos sujeitos da cidade para a constituição de programas e políticas públicas que pretendem o direito à cidade e à materialização dos princípios da Carta das Cidades Educadoras 6 .

${ }^{6}$ A primeira carta das cidades educadoras, com os princípios básicos, foi elaborada no Congresso Internacional das Cidades Educadoras, em 1990, na cidade de Barcelona. Foi revista no II Congresso Internacional (Bolonha, 1994), no VIII Congresso (Génova, 2004) e em 2020, para adaptar as suas 
Acolhendo diferentes manifestações culturais como forma de expressão que anunciam pertencimento ou denunciam exclusão, a gestão busca dinamizar, de forma participativa, as ações com o entendimento de que as mesmas contribuem para a formação permanente dos sujeitos através dos processos de humanização propiciados pela arte em suas diferentes áreas.

Espaços públicos com a dimensão educativa são reconhecidamente potentes mecanismos que dinamizam as múltiplas concepções culturais. No conjunto do trabalho pedagógico da e com a cidade, os espaços públicos são fundamentais para a emancipação da população.

Nesse aspecto, Soledade contempla diferentes espaços educativos como a Praça Central Olmiro Ferreira Porto, que integra múltiplas manifestações culturais e curriculares como Feira do Livro, encontro de bandas marciais, aulas com diferentes temáticas, ensaios de dança gaúcha e folclórica, oficinas do Centro Cultural (capoeira, ballet, jazz ...); o Castelinho, organizado pela Academia Soledadense de Letras, em parceria com o poder público, o qual disponibiliza para a comunidade uma diversidade de obras literárias de escritores soledadenses e de outras regiões do estado e do país; a Pira da Pátria e Pira Farroupilha; Monumento ao Gaúcho, que homenageia grandes laçadores de Soledade que entraram para a história como campeões do Rodeio Internacional de Vacaria, é considerado um laboratório público de cultura e lazer dos múltiplos segmentos da comunidade.

Além desses, existe o Centro Cultural que integra o cenário de práticas sociais coletivas que sinalizam para o direito à cidade e aos bens materiais e imateriais enquanto patrimônio da comunidade, combatendo qualquer forma de discriminação e incentivando o acesso e a participação de todos nas diferentes formas de cultura. O referido Centro abriga o museu histórico-municipal e o arquivo, além da biblioteca pública e o departamento de cultura do município.

As oficinas culturais, coordenadas pelo centro cultural, são realizadas gratuitamente para a população e envolvem pessoas dos seis aos oitenta e três anos. As modalidades como teatro, coral, teclado, gaita, canto, violão, cultura gaúcha, declamação/poesia, chula, dança, artesanato, capoeira estão contribuindo para a promoção e valorização das diferentes formas de aprender juntos, de forma a valorizar as manifestações que coadunam com a humanização dos sujeitos.

perspectivas aos novos desafios e necessidades sociais. Os princípios o direito à cidade educadora, 0 compromisso da cidade e serviço integral das pessoas, integram o conjunto do documento. 
Dentre os eventos promovidos pelo departamento cultural, destacam-se os de cunho histórico-cultural, em que, além dos profissionais que participam da elaboração, a comunidade através dos pesquisadores locais, é acionada para que juntos busquem a fidelidade dos fatos. 0 elenco, formado por alunos e professores das oficinas do centro cultural, intensifica o trabalho e o cunho cultural, alicerçados pelo museu, arquivo histórico e biblioteca.

O Parque de Eventos Centenário Rui Ortiz configura-se por ser um dos mais belos do estado, muito usado para manifestações artísticas e culturais, como o ENART- Pré-Mirim, Mirim e Juvenil voltado a crianças e jovens; Rodeios Campeiros e Artísticos, Baile da Terceira Idade, espaço para ciclistas e atividades físicas diversas, sendo ainda um local de encontro de famílias e amigos nos finais de semana. É considerado um dos melhores parques do RS para a realização de eventos, pois dispõe de infraestrutura para acolher com qualidade diferentes atividades simultâneas.

Junto ao Parque de Eventos Rui Ortiz, localiza-se o Museu da Pedra e Mineralogia Egisto Dal Santo, inaugurado em 20 de maio de 2016, apresenta-se como mais um lugar consolidado como espaço cultural e de aprendizagem. Um atrativo espaço de turismo no qual proporciona conhecimento dos processos que envolvem a pedra, desde sua formação e história passando pelas diferentes etapas, como extração e mineração, beneficiamento e comercialização. O Museu é o ponto de partida da Rota Turística Caminhos Preciosos que leva o turista a conhecer, experimentar a gastronomia local, da Agricultura Familiar, saboreando deliciosos pães, cucas, doces, morangos com chocolate e apreciar as belíssimas paisagens naturais que existem na Rota.

Todos esses espaços educativos revelam processos e organização cultural de Soledade, intencionalidades e estratégias de gestão municipal. Essa rede de espaços públicos que fazem parte da cidade pode ser associada à Carta das Cidades Educadoras, princípio 10, do eixo "O compromisso da cidade" que sinaliza para o governo municipal "dotar a cidade de espaços, equipamentos e serviços públicos adequados ao desenvolvimento pessoal, social, moral e cultural de todos os seus habitantes, prestando uma atenção especial à infância e à juventude" (2004, p. 06). Requer observar que todo e qualquer espaço público adquire sentido na relação com os sujeitos da cidade que se formam e se transformam com o seu território. 


\section{CONSIDERAÇÕES FINAIS}

O desenvolvimento do presente estudo possibilitou uma análise da importância das raízes culturais do povo, no sentido da afirmação de sua identidade e pertinência à sua região. Além disso, no decorrer do presente trabalho, evidenciou-se que a cultura territorial influencia na formação da identidade deste povo por meio das instituições sociais constituindo uma Cidade Educadora.

No caminho das Cidades Educadoras, muitas outras ações, além das citadas neste trabalho, terão que existir para assegurar que Soledade continue fazendo parte da AICE. O território possui uma série de possibilidades de alternativas de aprendizagem- caldo de cultura comum, saberes locais e memórias das pessoas, as vivências, suas experiências para dentro de um diálogo com a Escola e a Universidade num processo contínuo em que é capaz de criar um currículo de Cidade Educadora.

No decorrer da pesquisa, voltamo-nos para a história, projetamos nosso olhar para 0 futuro e questionamos o potencial educativo desta cidade. A escola passa a se tornar referência, ponto de encontro, fomentação de práticas educativas junto à cidade. Com seu compromisso social, a cidade e a escola, ao se entrelaçarem, assumem papel transformador com as comunidades, realizando trabalhos em rede, movimentando o currículo em diálogo e articulação com sua comunidade, afirmando o potencial educativo com práticas inovadoras em projetos para a capacitação cidadã da população.

Vários desafios teremos pela frente, os investimentos de governos e políticas públicas para resolver as desigualdades sociais, desafiar que todas as pessoas se sintam responsáveis uma pelas outras, precisamos de uma sociedade ecologicamente correta, economicamente viável, socialmente justa e culturalmente diversa.

Nesse sentido, é fundamental reconhecer o espaço da cidade, como um espaço voltado para todos, construir uma cidade com a participação social, precisa de um projeto articulado e integrado no qual crianças, jovens e adultos se envolvam e sejam educados nos princípios de Cidade Educadora.

As ruas e outros espaços públicos, ainda hoje, apesar do advento de tecnologias, são espaços essenciais para a socialização e o encontro de todos. A cidade educadora necessita constituir ações intersetoriais, diálogos e movimentos entre as gerações, interdependência da escola com suas comunidades, permanente autoavaliação dos propósitos, princípios e ações, 
preservação e difusão da identidade, acolhimento à diversidade, fortalecimento do trabalho coletivo e promoção da cultura com a participação dos sujeitos da cidade.

O desafio é a reafirmação do direito à cidade, da curricularização dos espaços públicos, da construção coletiva de políticas públicas, da fomentação do sentimento de pertencimento, de aprender a cidade, com a cidade e na cidade, pois como disse Santos (2002) esse não é um movimento concluído, aliás, sua natureza é ser um movimento sem fim.

\section{REFERÊNCIAS}

ASSOCIAÇÃO INTERNACIONAL DAS CIDADES EDUCADORAS. Disponível em: https://www.edcities.org/pt/quem-somos/Acesso em:16 jun. 2021

BRANDÃO, Carlos Rodrigues. A Educação Como Cultura. 2.ed. São Paulo: Brasiliense, 2002. p.15 0 que é educação. São Paulo: Editora Brasiliense, 2007.

CABEZUDO, Alicia et al. Cidade Educadora - Princípios e Experiências. São Paulo:Cortez, 2004.

CRAIDY, Carmem. 0 enlaçamento do processo educativo com o projeto de cidade. Revista Fazeres e Saberes Educativos. Prefeitura Municipal de Getúlio Vargas. № 1, 2002.

FREIRE, Paulo. II Congresso Internacional de Cidades educadoras, Gotenburgo, Suécia, 25-27 novembro, 1992.

GADOTTI, Moacir. A escola na cidade que educa. Cadernos Cenpec. Nova série. v.1, n.1 (2006) Disponível:

http://cadernos.cenpec.org.br/cadernos/index.php/cadernos/article/view/160/189. Acesso em: 13 jun. 2020.

GOMÉZ-GRANELL, Carmen; VILA, Ignácio. A cidade como projeto educativo. Barcelona: Artmed, 2003.

LESSA, Barbosa .Tese "O Sentido e o Valor do Tradicionalismo". Santa Maria: [s.e.], 1954.

LEVINSKI, Eliara Z.; TASCHETO, Márcio. Cidade Educadora: um desafio para Passo Fundo. In. BARELLI, Cristiane et al (org). Caderno de educação socioambiental: projeto de extensão Goio-En "Saneamento para a vida". Passo Fundo: Ed. Universidade de Passo Fundo, 2013.

PARANÁ. Manual do Grêmio Estudantil. SEED, 2012. Disponível em http://www.alunos.diaadia.pr.gov.br/arquivos/File/gremio_estudantil/manualgremio.pdf. Acesso em: 13 jun.2020.

SANTOS, Boaventura de Sousa. Democratizar a democracia: os caminhos da democracia participativa. Rio de Janeiro: Civilização Brasileira, 2002. 